Oper Orthop Traumatol $2017 \cdot 29: 279$

DOI 10.1007/s00064-017-0504-x

Online publiziert: 19. Mai 2017

c) Springer Medizin Verlag GmbH 2017

CrossMark

\author{
A. B. Imhoff \\ Abteilung für Sportorthopädie, Klinikum rechts der Isar der TU München, München, Deutschland
}

\title{
Perigenikuläre Osteotomien
}

Achsenfehlstellungen an den Beinen sind seit Jahrzehnten eine Domäne der korrigierenden Orthopädie - sei es bei angeborenen oder erworbenen Fehlstellungen. Eine Fehlstellung des Beins führt im Kniegelenk durch die ungleichmäßige Beanspruchung zu Knorpelschäden und in der Folge zu Arthrosen. Mit der Achsenkorrektur kann die Beinachse verändert und der geschädigte Anteil eines Gelenks von zu viel Belastung bewahrt werden.

Mit den heute zur Verfügung stehenden winkelstabilen Implantaten, den sog. Plattenfixateuren, sind nun die Indikationsmöglichkeiten größer geworden. Wir können heute bereits bei jungen und sportlich aktiven Patienten frühzeitig die Achsen korrigieren und die Gelenkspalten symmetrisch belasten lassen.

Das Ziel heute ist, immer eine horizontale Belastungsachse des Kniegelenks zu erreichen, was durch eine exakte präoperative Planung mit den Planungssystemen, wie z. B. mediCAD erleichtert wird. Auf diese exaktere Planung geht der erste Beitrag von Dietrich Pape ein, der sowohl die Bildgebung, die bei der Planung notwendig ist, aber auch die präoperative Planung extensiv beleuchtet. Je nach Deformitäten im und ums Kniegelenk können nun auch minimal-invasiv sowohl am distalen Femur medial oder lateral, wie auch an der proximalen Tibia medial Korrekturen notwendig sein. Manchmal muss aus diesen Gründen eine Osteotomie auch als sog. 2-Etagen-Korrektur am distalen Femur und an der proximalen Tibia geplant werden. Die minimal-invasiven winkelstabilen Plattensysteme erlauben 2-Etagen-Korrekturen auch gleichzeitig mit früher Belastung.

Auch wenn die Verwendung der winkelstabilen Plattenfixateure eine frühe Belastung ermöglicht, erfordert die Be- handlung von Begleitpathologien, wie knorpelregenerative Therapien, Bandrekonstruktionen des vorderen und hinteren Kreuzbandes oder der Peripherie, oft eine längere Entlastungs- und Rehabilitationsphase. Dies gilt insbesondere bei der chronischen Patellarinstabilität, wo die Osteotomie begleitend in den Behandlungsplan zur Korrektur der ungünstigen Torsion einbezogen werden muss.

Wir hoffen, Ihnen mit diesem Schwerpunktheft ein Hilfsmittel an die Hand geben zu können, um mit den neuen Methoden eine exakte Planung für die perigenikulären Osteotomien zu ermöglichen. In diesem Sinne wünsche ich allen Lesern viel Vergnügen und bin für jede Art von Feedback dankbar.

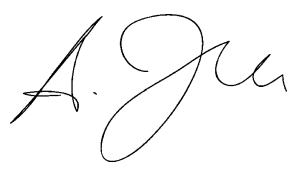

Andreas B. Imhoff, München

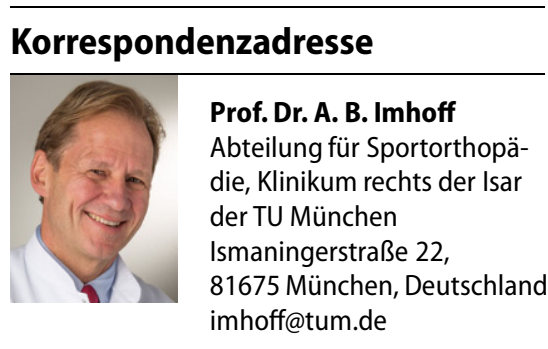

Interessenkonflikt. A.B. Imhoff: Berater bei der Fa. Arthrex, $\mathrm{GmbH}$, Freiham. 